Liverpool Preprint: LTH 371

Edinburgh Preprint: 96/5

hep-lat/9605025

21 May 1996

\title{
Orbitally excited and hybrid mesons from the lattice
}

\author{
UKQCD Collaboration \\ P. Lacock, C. Michael \\ Theoretical Physics Division, Department of Mathematical Sciences, University of Liverpool, \\ Liverpool, L69 3BX, U.K. \\ P. Boyle, P. Rowland \\ Department of Physics \& Astronomy, University of Edinburgh, Edinburgh EH9 3JZ, Scotland
}

\begin{abstract}
We discuss in general the construction of gauge-invariant non-local meson operators on the lattice. We use such operators to study the $P$ - and $D$-wave mesons as well as hybrid mesons in quenched QCD, with quark masses near the strange quark mass. The resulting spectra are compared with experiment for the orbital excitations. For the states produced by gluonic excitations (hybrid mesons) we find evidence of mixing for non-exotic quantum numbers. We give predictions for masses of the spin-exotic hybrid mesons with $J^{P C}=1^{-+}, 0^{+-}$, and $2^{+-}$.
\end{abstract}

\section{Introduction}

A quantitative determination of the hadronic spectrum of QCD has been one of the major goals of physics for the past 20 years or so. A way to determine the spectrum from first principles was first made possible in lattice gauge theory, and much effort has been invested to obtain the spectrum using lattice techniques.

Up until now, most effort has gone into the determination of the masses of states that are accessible through the use of spatially local operators, i.e. states with quantum numbers that can be constructed using quark and antiquark propagators from a common point. This is mainly due to practical considerations (e.g. limited computer-time resources). However, extending these studies to include states with excited orbital momentum $L$ is of importance in order to gain a more complete insight and better understanding of the QCD spectrum. 
From a theoretical point of view, the $L$-excited quark model gives a very good qualitative description of the observed meson spectrum. The low lying mesons are the $S$-wave $\pi$ and $\rho$ multiplets, next come the $P$-wave states: $b_{1}(B), a_{0}(\delta), a_{1}\left(A_{1}\right)$ and $f_{2}\left(f_{0}\right)$ multiplets, where we give the historic name of the isovector member of the multiplet in brackets. Some of the $D$-wave states are also recognisable: $\pi_{2}$ and $\rho_{3}(\mathrm{~g})$. This classification incorporates most of the prominent low-lying mesons. As the orbital angular momentum $L$ is increased, linear Regge trajectories are found with $m^{2}$ approximately linear in $L$. States which lie outside the naive quark model, glueballs and hybrid mesons, are not clearly seen in experimental analyses of the spectrum. It is important to understand these salient features of the meson spectrum directly from QCD. It is also necessary to guide experimental searches for hybrid mesons by establishing the non-perturbative QCD predictions for them.

Lattice gauge theory provides a way to achieve this. Indeed the static quark approximation has provided information on both the $L$-excitations and hybrid levels of heavy quark systems such as $c \bar{c}$ and $b \bar{b}$ mesons. This programme of study has been conducted in the context of the static quark potential. A determination of spin-spin and spin-orbit potentials has enabled the fine and hyperfine structure to be understood quantitatively. One of the successes has been the lattice identification [1] of the nature of the the long-range spin orbit potential which is responsible for the correct description of the splitting of the $P$ - and $D$-wave mesons. Another important input from lattice QCD has been the determination [2] of the ordering and energy of the hybrid levels. This approach uses the Schrödinger equation in the adiabatic approximation and the prediction is that hybrid mesonic states will lie close to the $D \bar{D}$ and $B \bar{B}$ thresholds. It is important for experimental searches to decide more accurately whether these hybrids are above or below these thresholds. This requires a determination for hybrid mesons of the effects of mixing with other levels and the effects of spin-orbit contributions. These are expected to be quite large and have not been evaluated by lattice methods. As well as the static quark lattice work described, the effective lagrangian of NRQCD would also be able to explore the hybrid spectrum for $b$-quark systems.

Here we wish to pursue these studies of spectroscopy for lighter quarks. This is of great experimental interest since the heavy quark results described above are only of qualitative relevance to lighter quarks. We shall proceed by using explicit quark propagators to construct mesonic states. Thus we shall directly obtain the masses of the mesons with the quantum numbers constructed. We show how to study all $J^{P C}$ states which allows an investigation of hybrid mesons in this way.

We make a first step by using the quenched approximation to explore these states from first principles. The quark mass used here is comparable to the strange quark mass. We are able to explore the $L$-excited spectrum for $P$ - and $D$-waves. We also explore hybrid mesons and look explicitly at states with $J^{P C}$ - values not allowed in the quark model. These exotic mesons are an important signal experimentally of gluonic excitations in the meson spectrum.

The $P$ - and $D$-wave excited mesons have been explored previously using either local operators 
(this only gives access to the $P$-wave $1^{+-}, 0^{++}$and $1^{++}$mesons) [3, 4, 5] or by using explicit non-local operators [6]. The work by DeGrand and Hecht [6] used Coulomb gauge fixing and explicit $P$ - and $D$-wave non-local operators which were motivated by the spherical harmonics. This pioneering study [6, 7] has been extended to include a classification of the $P$-wave states under the lattice symmetries [5]. Compared to previous work, we are able to get good signals at lower quark mass, we have reduced finite size effects and we are able to study a very wide range of states using the full classification under the symmetries of the lattice. The additional advantage of our approach is that we can study this wide range of quantum numbers with only two quark propagator inversions per configuration.

The motivation for the construction of our mesonic operators is to follow similar ideas to those found to be successful [2] for the analysis of the ground state potential and excited potential between static quarks. Even though we work with much lighter quarks (near the strange quark mass) we expect similar methods to be promising. Thus we use non-local meson operators which are gauge invariant with a path of links joining the quark and antiquark. A spatial fuzzing algorithm was found to be very efficient for improving the overlap with the ground state for static quark studies [8] and, moreover, using fuzzed links was found to be successful [9] for the construction of $S$-wave meson states. Hence we choose to use fuzzed links to construct the path here too.

The essence of our method is that at the source the quark is fixed at $x_{1}=(0,0,0,0)$ while the antiquark is fixed at $x_{2}=(0,0, r, 0)$. This implies that only two quark propagator inversions are needed. One of them is the usual local propagator from the origin and the other is just the same local propagator but from a local source shifted by $r$ in the 3-direction. The choice of path to join these sites is then quite flexible - no additional quark matrix inversions are needed for different choices. We find that a straight path is appropriate for a study of $P$ - and $D$-wave excitations, while a U-shaped path gives access to a study of hybrid mesons. We are then able to construct and measure correlations of these sources with operators at the sink that allow a wide range of quantum numbers to be studied. The construction and classification of these mesonic operators on a lattice is discussed in detail in the Appendix. In particular we show how, in principle, mesons with any $J^{P C}$ value can be studied using lattice methods.

In the next section we present our results for the $P$ - and $D$-wave excited mesons. We then discuss the hybrid meson spectrum and wavefunctions. In this work we have limited statistics so we concentrate on presenting the method and an analysis of the construction of the most efficient operators to use in such a study. We also give a first estimate of the mass spectrum using our methods. 


\section{Orbitally excited mesons}

Our numerical results for quenched $S U(3)$ gauge theory are obtained using quark propagators with local source at the origin and a further set of propagators in the same configurations with local source displaced by $r$ units from the origin in the 3-direction (the choice of spatial axes is arbitrary: one could just as well have chosen the 1- or 2- directions).

Both sets of propagators were calculated on a $16^{3} \times 48$ lattice at $\beta=6.0$ using a tadpole improved clover action [10]. In this exploratory study we use one hopping parameter value of $K=0.137$, which corresponds roughly to the strange quark mass. Thus our study directly bears on the $s \bar{s}$ spectrum. In this first study, we use 70 configurations and we set the source separation $r=6$. Our results will enable us to decide if this was a good choice of $r$. Since the static quark studies show that a large fuzzing level is optimum, we choose as our fuzzing level to use 18 iterations with a coefficient of the straight links $c=2.5$. We did explore using a smaller fuzzing level ( 5 iterations with $c=2$ ) and found that our results were essentially unchanged.

For the study of $S$-, $P$ - and $D$-wave states, we use straight paths to create the mesonic operators. For straight paths along the lattice axes, this allows a study of the states discussed in the Appendix. Here we choose to use the large component Dirac couplings to the quarks (i.e. $\left(1+\gamma_{4}\right) / 2$ projection). We follow the convention of using the historical name of the isovector meson to label the state we are discussing - although it is the strange iso-scalar that we are effectively creating. To explore the optimum choice of operator, we use straight paths of length $R$ at the sink and sum over all spatial sites to have zero momentum. The dependence of the mesonic correlation on $R$ gives direct information on the Bethe Salpeter wavefunction of the meson. This has already been studied by essentially our present methods for the $S$-wave mesons $(\pi$ and $\rho)[9,11]$.

To study the mesons most accurately, we use as many operators as possible in order to make the most constrained fit to the measured correlations versus $t$. Thus we can use results for several values of $R$ together. Moreover, we can include correlations from additional operators for those $P$-wave mesons which can be created by the small Dirac components of the quark bilinear (namely $\delta, \mathrm{A}_{1}$ and $\mathrm{B}$ ) together with an isotropic spatial operator. Since we have access to local-local and fuzzed-local correlations from 375 configurations 110, we are able to use these data to improve the mass determination substantially for those three mesons.

Because the correlations decrease rapidly with increasing time separation, we choose to fit to as low a $t$-value as possible with a two state fit (note that we only measured the shifted-source correlations up to $|t|=10$ ). Fitting simultaneously as many different types of correlation as possible, an accurate determination of the ground state mass is obtained since the approach to a plateau is well modelled. For the $\delta, \mathrm{A}_{1}$ and $\mathrm{B}$, we used $P$-wave operators with sizes $r=6$ at the source and $R=6$ and 2 at the sink, and $S$-wave operators using the small Dirac components with sizes $r=0$ and 6 at the source and $R=0$ at the sink. Thus we fit data from 7 different 
correlations of combinations of source and sink $P_{6} P_{6}, S_{0} P_{6}, P_{6} P_{2}, S_{0} P_{2}, P_{6} S_{0}, S_{0} S_{0}$ and $S_{6} S_{0}$. The effective masses for these correlations are illustrated for the $\mathrm{B}$ meson in Fig. 1. From the constraints of factorisation, we deduce that at least two excited states (ie a three state fit) would be needed to describe all the data down to $t=3$ because the $P_{6} P_{6}$ and $S_{0} S_{0}$ effective masses show excited state contributions which are not present for $S_{0} P_{6}$. Our fitting strategy is to determine the ground state mass by fitting the largest sample of data simultaneously, consistent with an acceptable estimate for $\chi^{2}$. This $\chi^{2}$ estimate comes from modelling the correlations among the data to be fitted by retaining the 8 largest eigenvalues and averaging the remainder [13]. The statistical errors on these fits are taken from a full bootstrap and a systematic error is estimated from varying the fit region in $t$, the data set fitted, and the type of fit. We illustrate in Fig. 1 a non-factorising fit with $t \geq 3$ for the $\mathrm{B}$ meson. Similar results for the ground state mass are obtained from a factorising fit with $t \geq 4$. The ground state mass values are shown in Table 1 .

For the $\mathrm{f}_{2}$ meson, we have access to it via two different cubic group operators. Fits to these operators separately show that they are consistent with having equal correlations. Our results are presented from fits assuming this. In this case only $P$-wave operators are available, so we use a simultaneous fit with $R=6,4$ and 2 . The ground state mass is given in Table 1 .

The results, shown in Fig. 2 and listed in Table 1, suggest that the $\mathrm{f}_{2}, \mathrm{~A}_{1}$ and $\mathrm{B}$ members of the $P$-wave multiplet are degenerate within errors. The $J=0$ state, $\delta$, is consistent with being lighter than the other $P$-wave states by $3 \sigma$ where, in order to investigate the fine structure splitting in the $P$-wave meson multiplet, we perform correlated $\chi^{2}$ fits to the data using the same bootstrap samples and $t$ interval $(3-10)$ - the results are shown in Fig. 2.

The $D$-wave mesons with $J^{P C}=2^{-+}$and $2^{--}$can be studied using the straight paths along the lattice axes. We make similar fits as for the $f_{2}$ meson (using two exponentials to fit simultaneously $R=6,4$ and 2). A typical fit (to the $2^{-+}$meson) is shown in Fig. 3. Our results for the masses using straight paths are shown in Table 1. However, the straight-path operator does not allow the $D$-wave mesons with $J^{P C}=2^{--}$and $3^{--}$to be separated. As discussed in the Appendix, L-shaped paths can be used to achieve this. We explored this using local sources at $(0,0,0,0)$ and $(6,0,6,0)$ and were able to determine the masses separately. The results, also shown in Table 1, from 20 configurations are consistent with these two states being degenerate within our errors.

For the vector meson, there are both $S$-wave and $D$-wave couplings. We made a simultaneous fit to the $S$-wave and $D$-wave operators (10 types of correlation in all of which the $S$-wave locallocal and fuzzed-local correlations come from 375 configurations) to determine the first excited state. The ground state was found to couple weakly to the $D$-wave operator whereas the excited state was found to couple strongly to the $D$-wave operator but weakly to the $S$-wave operator for $R=6$. The latter fact is a confirmation of our previous [9] observation that the excited state had a node at $R=6$. The systematic errors on the excited state determination are relatively large because the ground state dominates at large $t$. Our result with statistical errors only is given in Table 1. 


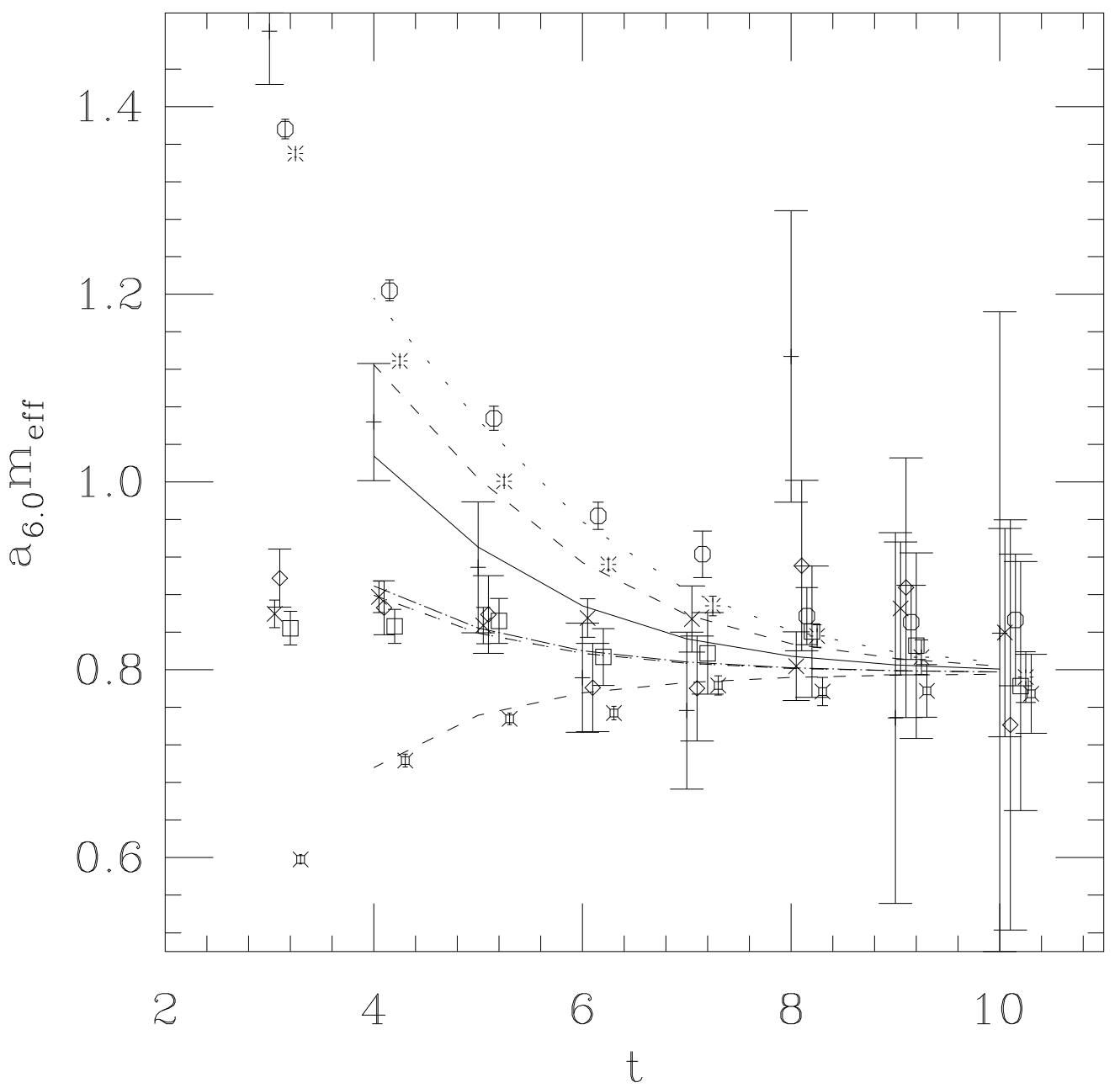

Figure 1: The two state fit to the effective mass for the $1^{+-}$meson versus time $t$. We used $P$ wave operators with sizes $R=6$ and 2 , and $S$-wave operators using the small Dirac components with sizes $R=0$ and 6 . Effective mass results shown are from correlations of combinations of source and sink $P_{6} P_{6}(+), S_{0} P_{6}(\times), P_{6} P_{2}(\diamond), S_{0} P_{2}$ (octagon), $P_{6} S_{0}(\square), S_{0} S_{0}\left(^{*}\right)$ and $S_{6} S_{0}$ (fancy square). 


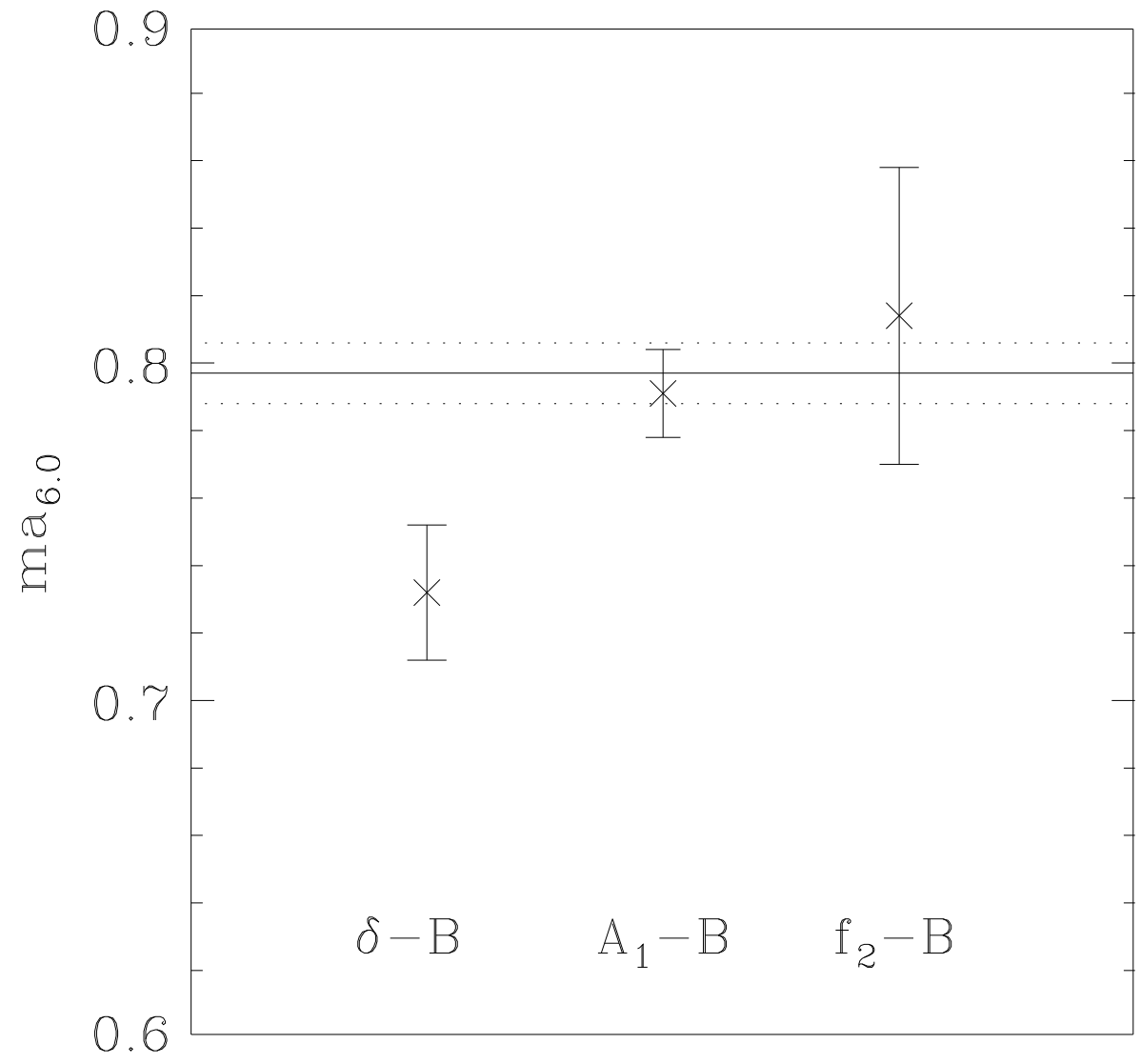

Figure 2: The differences of the mass (in lattice units) for the $P$-wave triplet states relative to the singlet. 


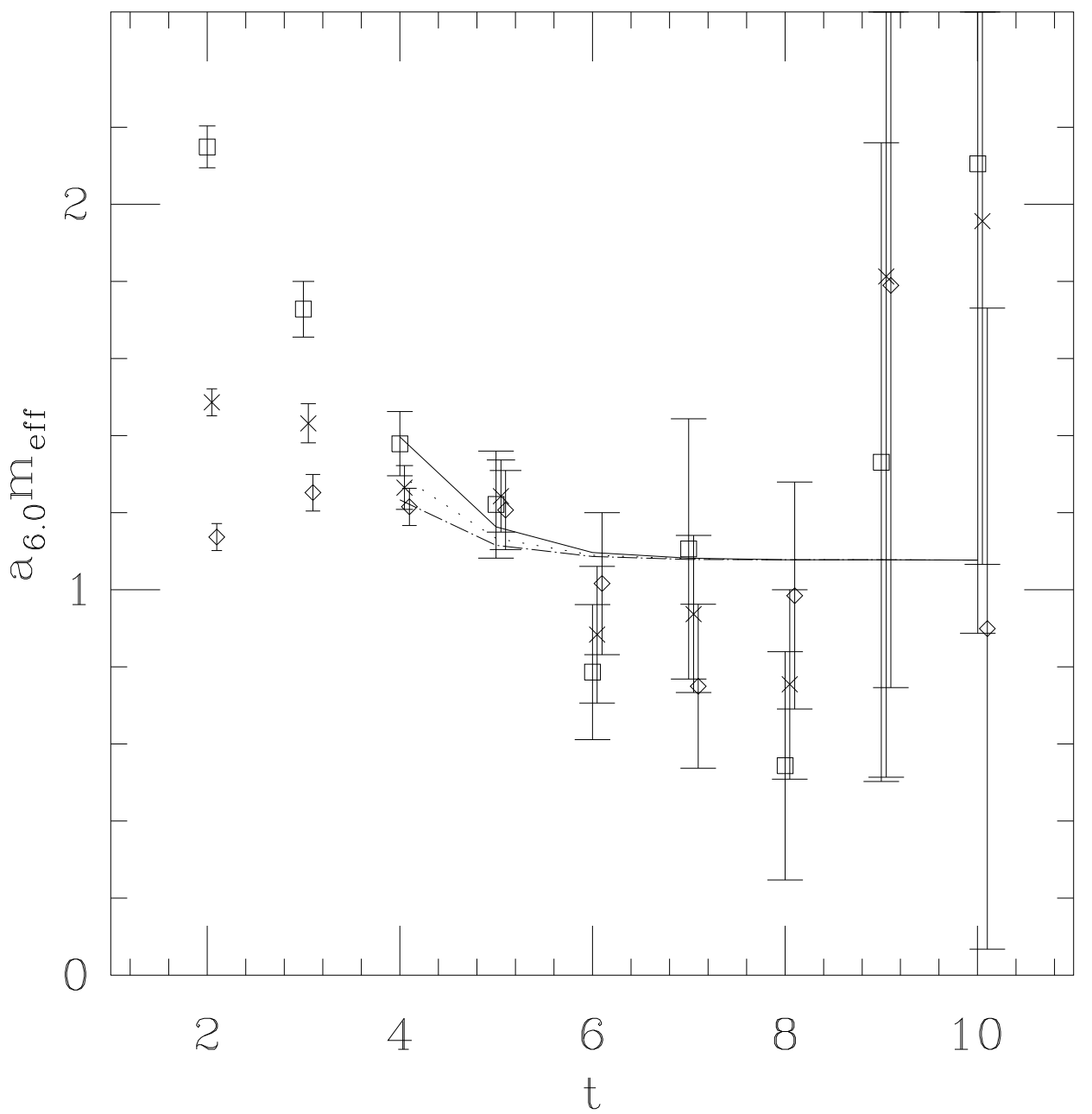

Figure 3: The two state fit to the effective mass for the $2^{-+}$meson versus time $t$. Correlations for sink sizes $R=2(\diamond), 4(\times)$ and $6(\square)$ are shown, where the source has $r=6$. 


\begin{tabular}{|c|c|c|c|c|c|c|}
\hline & $\begin{array}{l}\text { meson } \\
\text { multiplet }\end{array}$ & $J^{P C}$ & t-range & $\begin{array}{l}\text { mass } \\
m a\end{array}$ & $\mathrm{~m} / \mathrm{m}(\phi)$ & $\begin{array}{c}\operatorname{expt}(s \bar{s}) \\
\mathrm{GeV}\end{array}$ \\
\hline \multicolumn{7}{|l|}{$L=0,4$} \\
\hline & $\pi$ & $0^{-+}, 4^{-+}$ & 5,23 & $0.413(1)$ & $0.76(1)$ & 0.68 \\
\hline & $\rho$ & $1^{--}, 3^{--}$ & 5,23 & $0.540(2)$ & 1.00 & 1.02 \\
\hline \multicolumn{7}{|l|}{$L=1,3$} \\
\hline & $b_{1}(B)$ & $1^{+-}, 3^{+-}$ & 3,10 & $0.797(8)(10)$ & $1.48(2)$ & 1.42 \\
\hline & $\mathrm{a}_{0}(\delta)$ & $0^{++}, 4^{++}$ & 3,10 & $0.737(16)(10)$ & $1.36(3)$ & 1.21 \\
\hline & $a_{1}\left(\mathrm{~A}_{1}\right)$ & $1^{++}, 3^{++}$ & 3,10 & $0.789(8)(10)$ & $1.46(2)$ & 1.43 \\
\hline & $\mathrm{f}_{2}$ & $2^{++}, 3^{++}$ & 3,10 & $0.817(37)(30)$ & $1.51(7)$ & 1.52 \\
\hline & $\mathrm{f}_{2}$ & $2^{++}, 4^{++}$ & 3,10 & $\ldots$ & .. & $\ldots$ \\
\hline \multicolumn{7}{|l|}{$L=2,4$} \\
\hline & $\pi_{2}$ & $2^{-+}, 4^{-+}$ & 3,10 & $1.07(15)(15)$ & $1.98(28)$ & 1.81 \\
\hline & $\rho$ & $1^{--}, 3^{--}$ & 3,10 & $0.543(3)$ & $1.01(1)$ & 1.02 \\
\hline & $\rho^{\prime}$ & & & $1.25(13)$ & $2.31(24)$ & 1.68 \\
\hline & $\rho_{2}, \rho_{3}$ & $2^{--}, 3^{--}$ & 3,10 & $1.19(6)$ & $2.20(11)$ & \\
\hline \multicolumn{7}{|c|}{$L=2,3$} \\
\hline & $\rho_{3}(\mathrm{~g})$ & $3^{--}, 5^{--}$ & 2,8 & $0.86(19)$ & $1.59(35)$ & 1.85 \\
\hline & $\rho_{2}$ & $2^{--}, 4^{--}$ & 2,8 & $0.79(12)$ & $1.46(22)$ & \\
\hline
\end{tabular}

Table 1: The masses of the mesons with $S$-, $P$ - and $D$-wave excitations (with $s \bar{s}$ quarks but labelled by the name of the $\mathrm{I}=1$ particle in the multiplet). The $J^{P C}$ values are the lowest two values allowed by the lattice cubic symmetry. The angular momentum $L$ of the operator is shown similarly. For those $P$ - and $D$-wave mesons which are comparatively well determined in mass, the second error shown is an estimate of the systematic error from different fits. The last two rows are from 'diagonal straight paths' as described in the text (with lower statistics). More details of the construction of the operators used are given in the Appendix. The experimental masses of the $s \bar{s}$ states are given, as an educated guess in some cases (see Sect. 4).

Our results for $P$ - and $D$-wave mesons are considerably more accurate than those obtained by ref [6] for light quarks. Indeed, for the first time, we are able to see a signal for the splitting of the $P$-wave multiplet.

The extraction of the ground state as outlined above also allows the wavefunction to be obtained as $R$ is varied. Results are given for the $S$-, $P$ - and $D$-wave spin-singlet states in Fig. 4. These show clearly the expected functional behaviour with $R$ of $R^{L}$ for $S-, P$ - and $D$-wave mesons. Our use of a gauge-invariant path description allows us to probe this wave function out to beyond half the spatial lattice size - unlike the case where a gauge-fixed method is used [7] - results for $R>6$ were obtained with less statistics (20 configurations) and show a plausible behaviour with no disruption from finite spatial size effects.

Wavefunctions for the $P$ - and $D$-wave mesons have been obtained by DeGrand and Hecht [6]. 


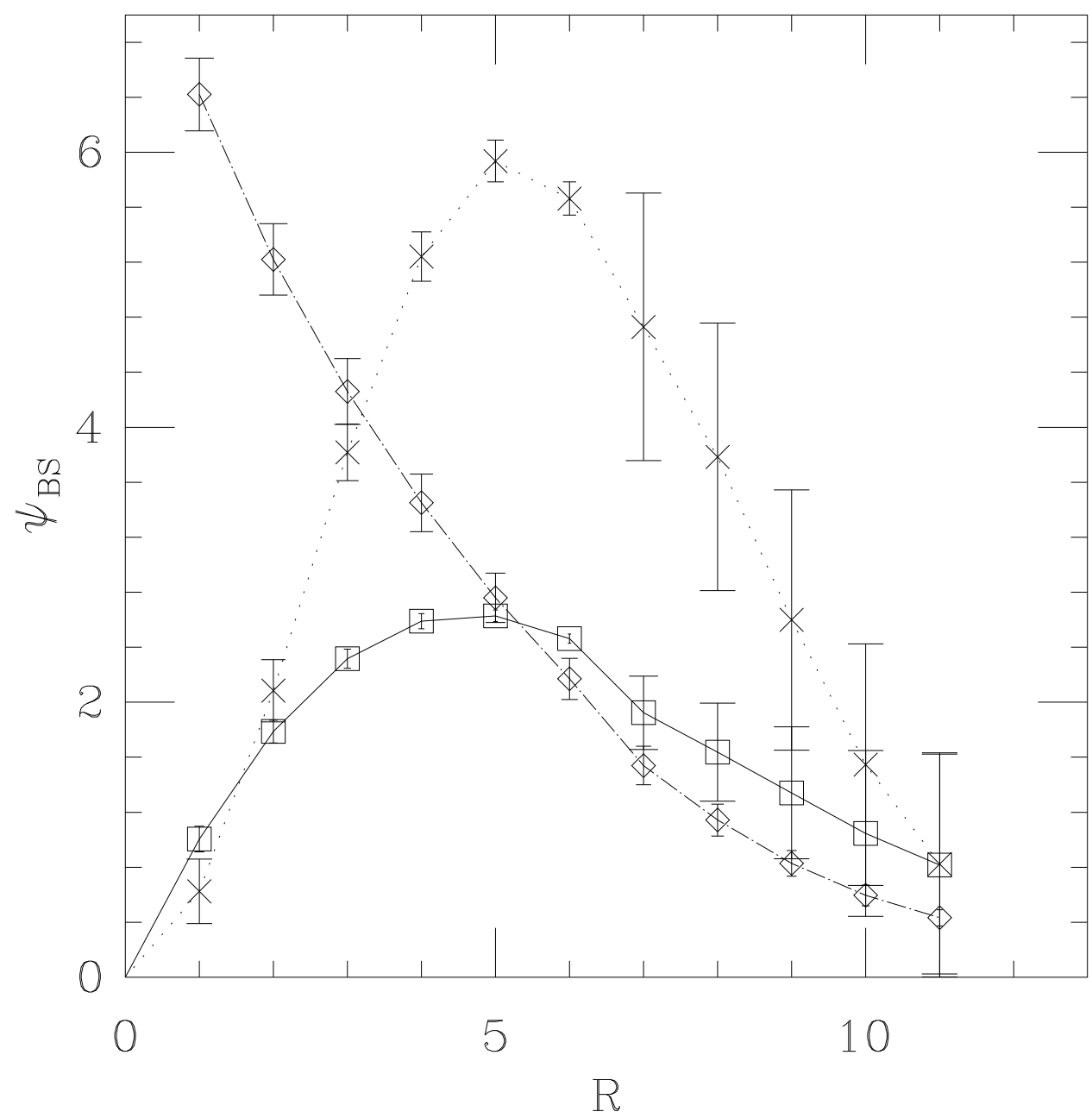

Figure 4: The Bethe Saltpeter wavefunctions obtained from our fuzzed straight paths of length $R$ for $S$ - $(\diamond), P-(\square)$ and $D$-wave $(\times)$ spin singlet mesons. The results for $R>6$ have smaller statistics. Here $R$ is in lattice units $\left(a \approx 0.5 \mathrm{GeV}^{-1}\right)$. The normalisations of the wavefunctions are arbitrary. 
The Bethe Salpeter wavefunction depends on the method used to construct it. Their method uses a Coulomb gauge fixing and so is not directly comparable to our determination. Qualitative features are in full agreement, however. The $D$-wave $\rho$ operator has also been studied [11] by a method similar to ours and the result is consistent.

Our choice of $r=6$ to use as a source separation is seen to be a reasonable compromise for the studies at different orbital momentum $L$. The original choice of $r=6$ was motivated by the discovery that the radial excitation of the $S$-wave mesons has a node at this value [9]. The orbital excitations have no such node as we see in Fig. 4. Since we measure correlations for different $R$ at the sink, we can construct optimum operators (in a variational sense that any excited state is minimised) at the sink.

Our choice of source operator is a 'white source'. It has contributions to nearly all quantum numbers. This is an advantage in allowing a comprehensive study of the meson spectrum without extra quark matrix inversions. It is a disadvantage, however, in that the signal in any given quantum number will be noisier because of the poor overlap of the source operator with that quantum number. This dilemma was discussed by DeGrand and Hecht [6] who concluded that a rather high symmetry was needed at the source. We find that the fuzzed construction allows our rather 'white' source to give a reasonable signal to noise ratio. Of course it could be improved for any particular state by using a quark propagator from a more symmetric fixed source (e.g. a suitable sum/difference of sources at $(0,0, \pm r),( \pm r, 0,0)$ and/or $(0, \pm r, 0))$. The increase in signal to noise would be compensated by decreased flexibility since only specific quantum numbers would be created. A compromise is possible, namely retaining flexibility by using inversions from several sources separately so that they can be combined with different coefficients as needed. However, because propagators from sources that are near to each other are strongly correlated to each other, it may be more efficient to increase the number of configurations studied rather than construct such multiple sources.

\section{Hybrid mesons}

The lowest lying hybrid levels for static quarks [2] come from an interquark potential in which the colour flux from quark to antiquark is excited in a transverse spatial plane as $\sqcap-\sqcup$ (cf. Appendix for notations). Excitations of this kind are clearly non-trivial gluonic contributions and the mesonic states in such excited potentials include exotic $J^{P C}$ values. As discussed in more detail in the Appendix, the same states can be accessed using propagating quarks by using a non-local mesonic operator in which the quark and antiquark are joined by such a difference of paths. This construction has the advantage that it does not require any more quark propagator inversions than those needed in the above study of $P$ - and $D$-wave mesons. Indeed, since the U-shaped paths can be transverse in either of two spatial directions, we have more operators available. Using eigenstates of the lattice symmetry at the sink, we are able to explore a wide 
range of quantum numbers of interest.

We first address the issue of creating optimum operators (in the sense of best signal to noise for the ground state of a given quantum number). Even with our fixed choice of $r=6$ in the 3 -direction as source separation, we can vary the transverse extent $d$ of the U-shaped path at the source and we can explore at the sink a range of values of $R$ and $D$ describing the U-shaped paths there. We expect the signal to increase with the area $(R \times D)$ of the U-shaped path but the noise will increase too. The static quark results [2] suggest that the hybrid mesons have very extended wavefunctions in the $R$-direction while the best signal comes from $D \approx 2$ for $R=6$. Another clue is that, for $S$-wave mesons made from propagating quarks, a smaller sink extent gives a less noisy signal than a smaller source size.

To investigate the optimum operators, we used the exotic state with $J^{P C}=1^{-+}$as a probe and varied the source and sink operators to maximise the signal to noise ratio at $t=2$ and 3. There is an advantage in using the same operator at source and sink because the effective mass is strictly an upper bound in this case. The best signal (i.e. lowest effective mass within errors from the ratio of $t=3$ to 2 ) for the effective mass for such a diagonal case came with $(r, d)=(R, D)=(6,6)$ although the $(6,3)$ case was very similar. For off-diagonal correlations, the optimum signal to noise for the correlation at $t=3$ was for $(r, d)=(6,6)$ and $(R, D)=(1,1)$. Taking into account the above, we consider the combinations $d=6 ;(R, D)=(6,6),(3,3)$ and $(1,1)$ in the analysis below.

Since the construction of correlators involving the hybrid meson operators can be quite computer-time intensive, we choose to include only the large components of the Dirac propagators, that is we effectively multiply the correlators with a factor of $\left(1+\gamma_{4}\right) / 2$ (cf. Appendix). Compared to the results obtained in the standard case of summing over all four components of the Dirac spinor we find that the signal, and thus both the correlation averages and errors, are smaller, but the physical results (e.g. ratios like the effective mass) are consistent in the two cases. However, even though there is no gain in statistical accuracy, the resulting speed up of the analysis code is quite substantial. Similar behaviour is found for the $P$ - and $D$-wave case.

For the hybrid operators we fitted simultaneously the three sink sizes discussed above and found acceptable fits for the $t$-range 2-10, using two exponentials. To stabilise the fits we fixed the mass difference to the first excited state at 1.5 in lattice units. Statistical errors were estimated by jackknife. To establish the systematic errors to the ground state masses determined by the fits, we varied fits by allowing the minimum $t$-value to be 3 or by allowing the first excited state mass to be free. In Figs. 5-7 we show the effective mass plot for the $J^{P C}=1^{-+}, 0^{+-}$and $2^{+-}$ exotic states with the fits. Note that the mesonic correlation data was fitted directly (accounting for the correlations among data points in the same way as for the fits to the orbital excitations) rather than the effective mass, which is plotted. The effective mass plot tends to mis-represent the errors when the errors are large. Even though the error bars are indeed quite large, the fits indicate that an acceptable plateau is reached for the $t$-interval considered. The results for the ground state masses obtained from the fits for the hybrid states are summarised in Table 2 and 


$\begin{array}{llccl}L=1^{+-} & \begin{array}{l}\text { meson } \\ \text { multiplet }\end{array} & J^{P C} & \text { t-range } & \begin{array}{l}\text { mass } \\ m a\end{array} \\ & & & & \\ & & 1^{--}, 3^{--} & 2,10 & 0.53(9) \\ \pi & 0^{-+}, 4^{-+} & 2,10 & 0.40(2) \\ & \pi_{2} & 2^{-+}, 4^{-+} & 2,10 & 1.56(76) \\ \pi_{2} & 2^{-+}, 3^{-+} & 2,10 & 1.40(53) \\ & \hat{\rho} & 1^{-+}, 3^{-+} & 2,10 & 0.99(28) \\ & & & & \\ & \mathrm{A}_{1} & 1^{++}, 3^{++} & 2,10 & 0.99(56) \\ & \mathrm{a}_{0} & 0^{+-}, 4^{+-} & 2,10 & 1.05(18) \\ & \hat{\mathrm{a}}_{2} & 2^{+-}, 4^{+-} & 2,10 & 1.29(44) \\ \hat{\mathrm{a}}_{2}, \mathrm{~b} 3 & 2^{+-}, 3^{+-} & 2,10 & 1.17(26) \\ & \mathrm{b}_{1}(\mathrm{~B}) & 1^{+-}, 3^{+-} & 2,10 & 0.84(31)\end{array}$

Table 2: The masses of the hybrid mesons created by operators with spatial excitation $L^{P C}=$ $1^{+-}$and $1^{-+}$. The $J^{P C}$ values are the lowest two values allowed by the lattice cubic symmetry. More details of the construction of the states are given in the Appendix. The masses are given in lattice units and are appropriate to the $s \bar{s}$ states

Fig. 8. The systematic errors on the ground state masses are consistent with being smaller than the quoted statistical errors.

The spin-exotic mesons cannot mix with $q \bar{q}$ mesons and so the ground state masses will be relevant to spectroscopy. The lattice values we find all have large errors and are compatible with being equal. The mass in physical units corresponds to 1.9(4) GeV. A further increase in precision will follow from the increase in statistics planned. Even our present statistical sample allows some estimate of possible splittings among these exotic states. The commonly held view is that the $J^{P C}=1^{-+}$state will be the lightest exotic and our results are consistent with this.

In principle, states with the same $J^{P C}$ and different internal spatial structure will mix in quantum field theory. Here we are able to explore these mixing possibilities in full by looking directly at the meson spectrum with propagating quarks. Fig. 8 clearly shows that mixing of the hybrid states with non-hybrid ones for vector and pseudoscalar mesons is quite significant. Indeed our hybrid operators for these states have a substantial overlap with the $q \bar{q}$ ground state mesons. The quantitative strength of this mixing is related to the lattice operator construction we use. Relating this to Minkowski wavefunction mixing is not straightforward. The subjective impression, however, is that the mixing is larger that expected. Moreover, in the case of the ground state vector meson (i.e. $\phi$ ), we can compare the surprisingly small mixing of the $D$-wave operator found above with the large mixing of the hybrid operator found here. This suggests that gluonic excitations mix more readily than orbital excitations. 


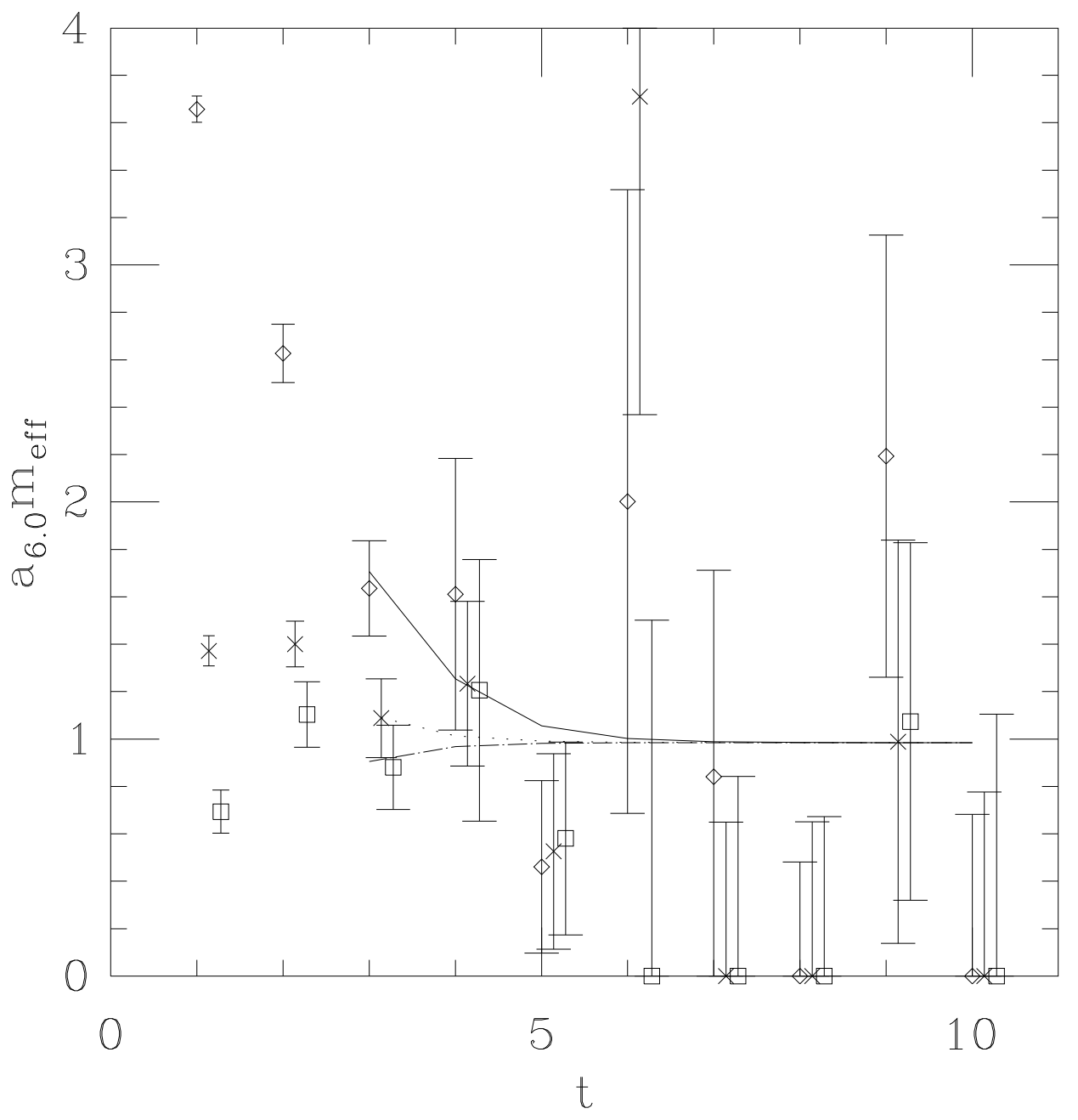

Figure 5: The lattice effective mass for the $J^{P C}=1^{-+}$hybrid meson versus time separation $t$. The source used was a U-shaped path of size $6 \times 6$, while the sinks were combinations of U-shaped paths of size $6 \times 6(\diamond), 3 \times 3(\times)$ and $1 \times 1(\square)$. The two state fit is shown. 


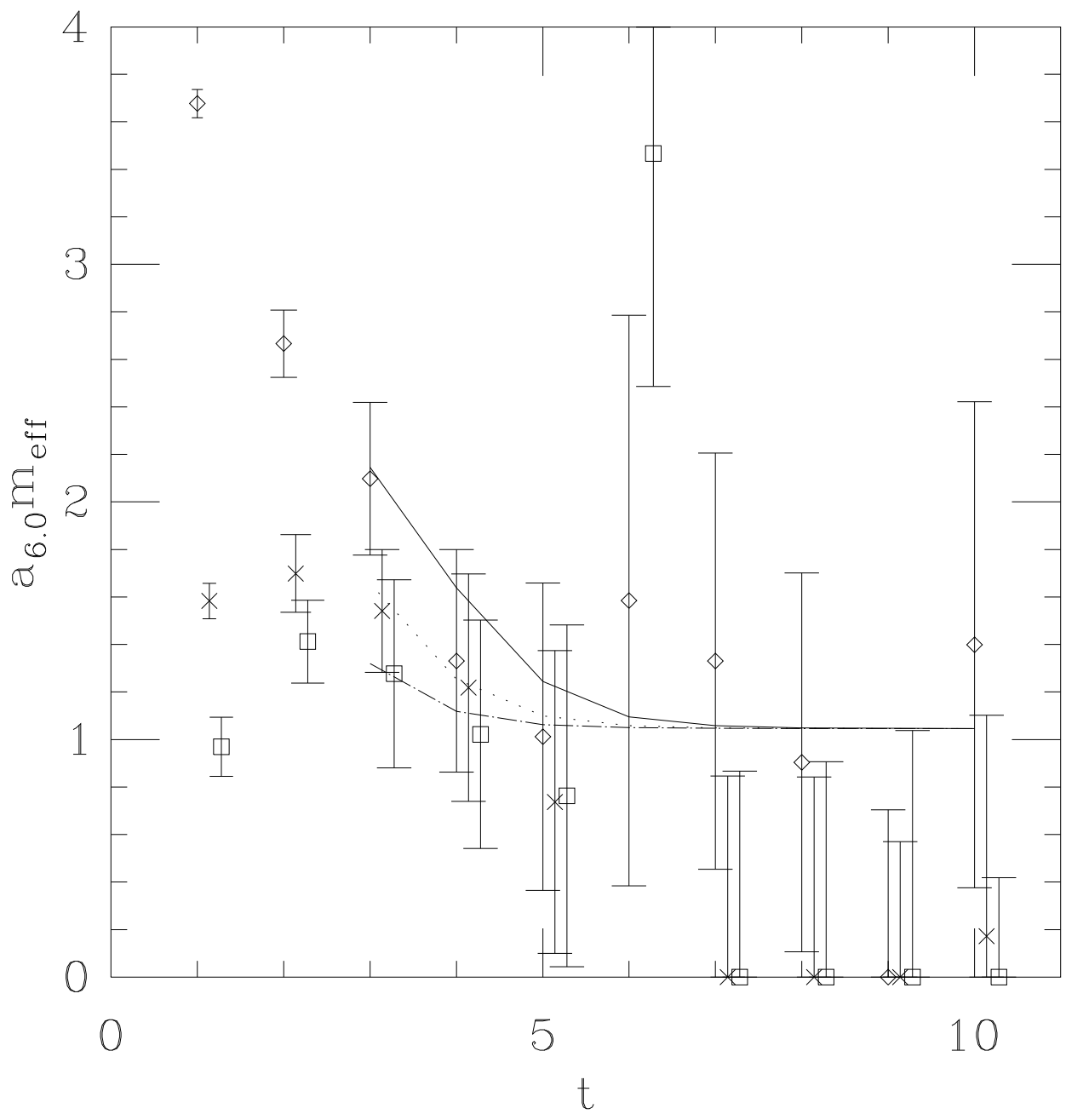

Figure 6: The lattice effective mass for the $J^{P C}=0^{+-}$hybrid meson versus time separation $t$. Other details as Fig 5 . 


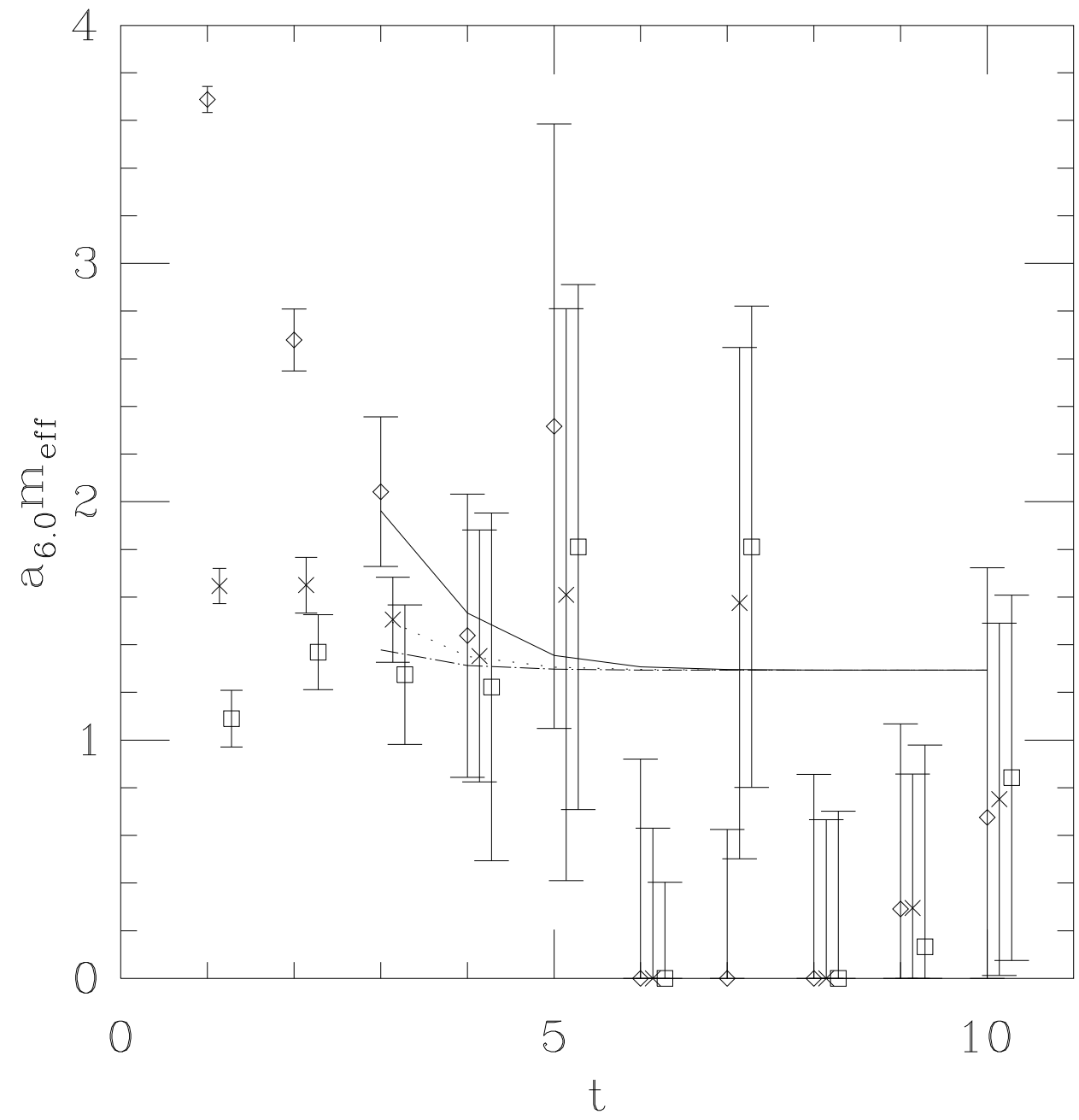

Figure 7: The lattice effective mass for the $J^{P C}=2^{+-}$hybrid meson versus time separation $t$. Other details as Fig 5 . 


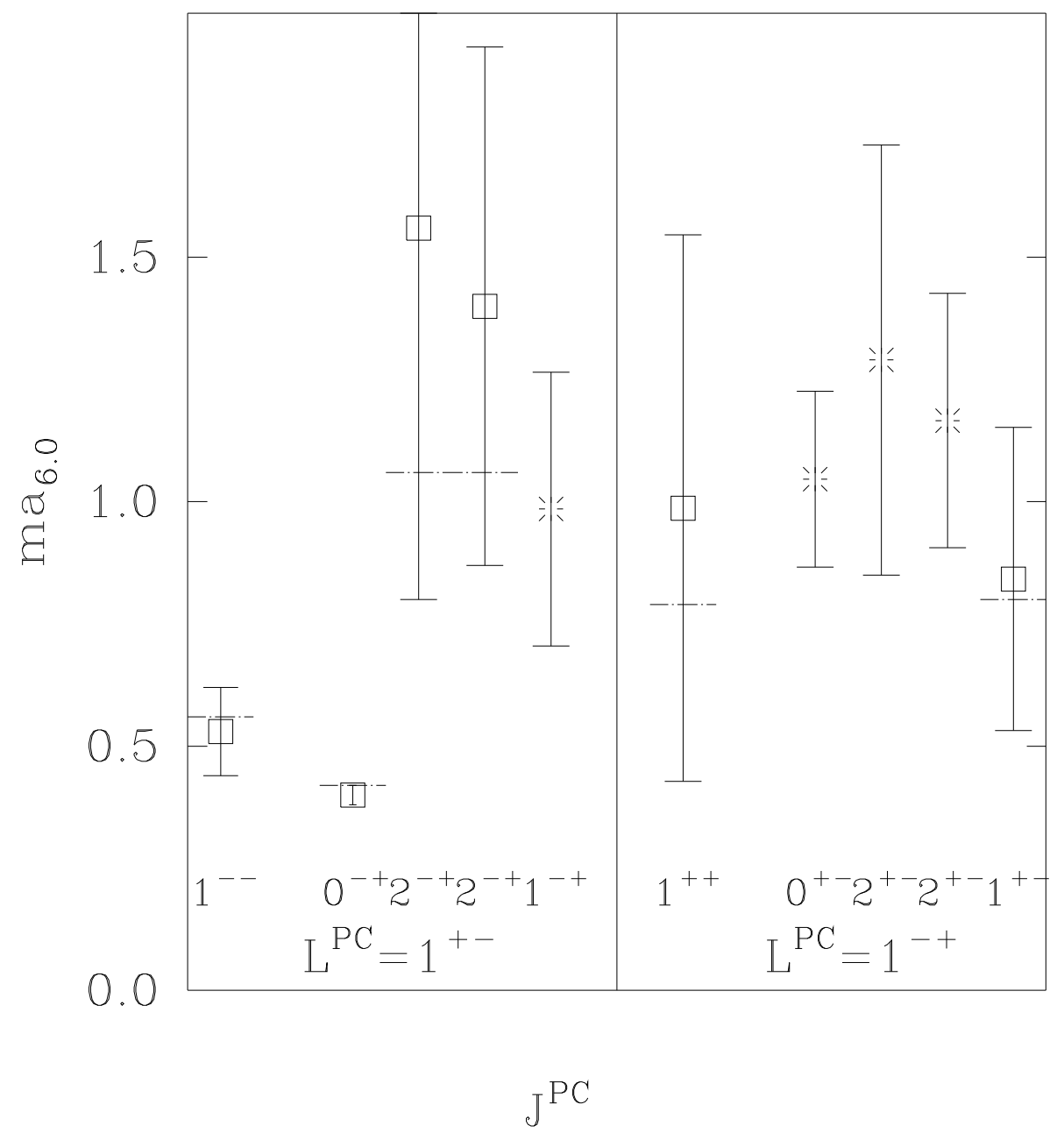

Figure 8: Results for the ordering of the hybrid meson levels for strange quarks. The states with burst symbols are $J^{P C}$ exotic. The dashed lines represent $L$-excited quark model states as determined on the lattice. The strong mixing of the states created by our hybrid operators with these is apparent for the pseuodoscalar and vector meson cases. 
The $0^{+-}$exotic meson can be studied in principle using an isotropic spatial operator with a $\gamma_{4}$ coupling between quark spins. With our larger statistics of 375 configurations for local-local and fuzzed-local meson correlations, we explored this using $\gamma_{4}$ at source and sink but found no signal. An investigation of correlations with a hybrid operator at the source (from 20 configurations with L-shaped paths) and a $\gamma_{4}$ sink also showed no clear signal with this statistical sample. These negative results could be explained if the spin-exotic state was indeed a hybrid with excited gluonic fields and so would have a small overlap with the isotropic operator considered here which has the gluonic fields in their ground state.

We have also investigated the possibility of using a gluon flux loop to construct the meson operator with an excited gluonic component, but with the quark and antiquark at the same site. This would allow correlations involving the operator to be explored using local propagators from one site only, which avoids the need for extra shifted-source inversions when studying hybrid states. The gluon flux loop should have similar features to that used in the heavy quark case for a static adjoint source [12]: for example an anti-symmetrised clover shape (i.e. clover paths minus their hermitean conjugate) which has the quantum numbers corresponding to a magnetic gluon $L^{P C}=1^{+-}$. The electric gluonic excitation can be created easily too - see ref [12]. Thus, using different gamma matrix combinations, all hybrid states can, at least in principle, be produced at source and sink. We studied several cases and were able to extract a signal from such closedloop gluonic excitations for a vector meson treated as a hybrid (using a closed magnetic gluon

loop with singlet quark spin combination). However, since we have already found large mixing between hybrid operators and the vector meson ground state, this case does not confirm that, in general, such a closed loop gluonic excitation is a useful way to create hybrid states. This lack of success is perhaps a consequence of the hybrid wavefunction being small for quark-antiquark separation of zero - as is known to be the case for heavy quarks [2].

\section{Discussion}

The mesonic spectrum in the quenched approximation has only been studied in detail on the lattice for some of the lowest states. Here we extend this study to considerably more states. The quenched approximation does not include effects from mixing of the $q \bar{q}$ system with $q \bar{q}$ of different flavour quarks, with $q \bar{q} q \bar{q}$ or with purely gluonic components. Thus a 'magic mixing' is expected in the quenched approximation with the $s \bar{s}, s \bar{q}$ and $q \bar{q}$ members equally split in $m^{2}$, where $q$ means $u$ or $d$. For the $J^{P C}=1^{--}, 2^{++}$and $3^{--}$mesons, such a mass spectrum is close to that observed and we compare our results with the $\mathrm{I}=0$ state assigned as $s \bar{s}\left(\phi, \mathrm{f}_{2}^{\prime}\right.$ and $\phi_{3}$ respectively).

Within the quenched approximation, one can study disconnected quark loops to estimate the strength of the mixing efects. For the pseudoscalar mesons, this approach [14] gives a reasonable explanation of the $\eta$ mass. Because these disconnected loop contributions affect $\mathrm{I}=0$ only, we 
estimate the mass of the pseudoscalar $s \bar{s}$ state as $0.68 \mathrm{GeV}$, assuming magic mixing, from the experimental masses of $\mathrm{I}=1$ and $\mathrm{I}=1 / 2$ states.

Charge conjugation $\mathrm{C}$ is not a good quantum number when the quark and antiquark have different masses. This implies that, for the axial and $2^{-}$mesons, the strange partners with $\mathrm{C}= \pm 1$ mix. For the $1^{++}$meson we identify the $s \bar{s}$ state as $\mathrm{f}_{1}(1427)$ while for the $1^{+-}$meson we use the ansatz that $M_{s \bar{s}}^{2}-M_{q \bar{q}}^{2} \approx 0.5 \mathrm{GeV}^{2}$ to set the $s \bar{s}$ state at $1.42 \mathrm{GeV}$ using the observed $\mathrm{b}_{1}$ mass. Similarly we estimate the $s \bar{s}$ state for $2^{-+}$at $1.81 \mathrm{GeV}$ using the observed $\pi_{2}$ mass.

For the scalar mesons, the experimental situation is complex - possibly owing to mixing with glueballs and meson-meson thresholds. As an estimate of the $s \bar{s}$ mass we again use the prescription of adding $0.5 \mathrm{GeV}^{2}$ to the $\mathrm{a}_{0}$ mass squared yielding an estimate of $1.21 \mathrm{GeV}$.

We have presented an estimate of the experimental spectrum for $s \bar{s}$ quarks. We now need to justify that this is appropriate for our lattice study. We measure the vector to pseudoscalar mass ratio as $M_{V} / M_{P}=1.31(1)$ and use the value of $J=\frac{1}{2} d M_{V}^{2} / d M_{P}^{2}$ to relate this ratio to the experimental $\mathrm{K}^{*}$ to $\mathrm{K}$ mass ratio. The average quark mass in a pseudoscalar meson is then assumed to be proportional to $M_{P}^{2}$. Values of $J$ from quenched lattices [4] are near 0.37 , while the observed meson spectrum yields values of $0.44-0.48$. This uncertainty leads to an estimate of our quark mass as 1.3 to 1.5 times the strange quark mass. We present our results as ratios to the vector meson mass and the systematic error from treating our quark mass as that of the strange quark is quite small for the $L$-excited and hybrid mesons compared to other sources of error.

Since the experimental states are for full QCD, they include decay effects which may also shift the mass somewhat from the quenched value. Some evidence for departures of the quenched meson spectrum from that of experiment has already been claimed [i] from studying the quark mass dependence. Here we are able to extract masses for a wide range of mesons. In this initial study, we use a fixed lattice spacing so we are unable to extract continuum limits. Nevertheless, the splittings between similar states (such as the $P$-wave mesons) would be expected to be reliably given at our lattice spacing. We work with quarks of fixed mass (close to the $s$ quark mass) and present our lattice results as ratios of masses to the vector meson mass $(\phi)$. As shown in Table 1, our overall spectrum is close to that of experiment (as interpreted above - see Table 1). We now discuss various topics in more detail.

We are able, for the first time, to measure a non-zero mass difference among the $P$-wave states: the $J=0$ member of the spin-triplet is found to be lighter. The sign of this splitting is the same as that found experimentally for $c \bar{c}$ mesons where the $J=0$ state is significantly lighter than the other $P$-wave states. The magnitude of the splitting of the $0^{++}$meson from the centre of gravity of the $P$-wave mesons is somewhat smaller than our estimate from experiment for $s \bar{s}$ quarks. As discussed above, the experimental scalar meson spectrum is difficult to interpret and our quenched results can act as a guide to phenomenological quark models. 
For the $D$-wave states, we are able to estimate the masses but with quite large statistical and systematic errors. The mass values we obtain appear to be somewhat higher than those expected experimentally. Bearing in mind the need to extrapolate to $a=0$, the corrections could be large since, at our lattice spacing, $m a>1$ for these states. Another possible problem is that, since $m a$ is large, the signal disappears quickly with increasing $t$ and so excited state contributions may not be completely removed. Our method does, however, enable us to get meaningful signals and a route to more precision is available using higher statistics and a smaller lattice spacing.

Previous to this work, the only lattice determination of the hybrid meson spectrum has been that obtained [2] in the static limit of the quenched approximation. The static quark analysis showed that the lowest lying gluonic excitation of the potential $V(R)$ has the symmetry $\sqcap-\sqcup$. Assuming the adiabatic approximation, the bound states in this potential will have a lowest excitation energy with one unit of angular momentum about the quark - antiquark separation axis. These excitations then correspond to $L^{P C}=1^{+-}$and $1^{-+}$spatial wavefunctions and are degenerate in energy, as described in the Appendix. Thus all eight hybrid mesons would be degenerate in this approach.

Within the static quark framework, this degeneracy will be broken by spin-orbit effects which shift the spin-exotic levels and cause mixing of the non-exotic levels with ordinary $q \bar{q}$ states. One clue to the sign of this effect for static quarks comes from considering the limit of zero separation. In this case of $r=0$, the symmetry classification of the gluonic states is different from that for $r \neq 0$, and the energies of these states have also been measured on a lattice 12 with the result that the $1^{+-}$gluonic mode is several hundred MeV lighter than the $1^{-+}$mode. This, in turn, suggests that hybrid states with the $1^{+-}$spatial wavefunction are likely to be lighter: so that $J^{P C}=1^{-+}$would be the lightest spin exotic hybrid.

We are able to explore this splitting of the degeneracy by a completely different method explicitly studying each state on a lattice using propagating quarks. We construct operators with $1^{+-}$and $1^{-+}$spatial wavefunctions, couple them to quark bilinears and measure the masses of the mesonic states. Our present results for the masses of the spin-exotic states are not sufficiently accurate to indicate the level ordering unambiguously. One theoretical clue is that as $r \rightarrow 0$, our $1^{-+}$spatial wavefunction decouples faster than the $1^{+-}$spatial wavefunction. This analysis at $r=0$ implies in turn that hybrid states with a $1^{+-}$gluonic mode are likely to be at lower energy, resulting in a splitting of the hybrid states with $J^{P C}=1^{-+}$being the lightest exotic meson, as argued above for the case of static quarks. Our results are consistent with this scenario.

We are also able to measure matrix elements between our hybrid operators and the ground state mesons for the non-exotic $J^{P C}$ states. Our conclusion is that the mixing is surprisingly strong. This would imply that hybrid levels with pseudoscalar and vector quantum numbers would be unlikely to exist unmixed in nature.

There have been several experimental claims for hybrid mesons - for a review see ref [15]. Our results suggest that non spin-exotic candidates may need re-appraisal since big mixing effects 
are possible. For the exotic mesons, the favoured candidate to lie lowest will have $J^{P C}=1^{-+}$ and several experimental hints of such states have been reported.

If our discussion of the quark mass dependence of states in the quenched approximation applies to hybrid mesons, then we may use the ansatz that $M_{s \bar{s}}^{2}-M_{q \bar{q}}^{2} \approx 0.5 \mathrm{GeV}^{2}$ where $q$ means $u$ or $d$. This suggests that hybrid mesons composed of $u$ and $d$ mesons will be approximately $150 \mathrm{MeV}$ lighter than the $s \bar{s}$ mesons for masses around $1.8 \mathrm{GeV}$. For strange mesons, the lack of $\mathrm{C}$ invariance will mask the experimental identification of spin exotic states.

We have presented methods which enable the meson spectrum to be fully explored for all $J^{P C}$ values using non-perturbative lattice methods. The results we have obtained show that accurate determinations of masses are possible by these methods. We intend to extend this approach by using more statistics, several quark mass values and a range of lattice spacings so as to get precise continuum masses in the quenched approximation.

\section{Acknowledgements}

We acknowledge support from EPSRC grant GR/K/41663 to the UKQCD Collaboration. We thank David Richards for his help in setting up the inversion codes for the shifted local propagators.

\section{A Appendix: Construction of mesonic operators.}

To study spectroscopy in lattice gauge theory, one needs operators at fixed time which create and destroy a mesonic state. By considering the correlation of such operators at time separation $t$ in the vacuum, the masses of the transfer matrix eigenstates can be extracted. We consider the construction of bosonic operators which contain a quark and an antiquark. The case we shall explore is where the quark is at $x_{1}$ and antiquark at $x_{2}$ and they are joined by a product of links along a path $\mathrm{P}$. This is a gauge invariant construction. The general form of the operator is then

$$
\bar{\psi}\left(x_{2}\right) \Pi_{\mathrm{P}} U \Gamma \psi\left(x_{1}\right)
$$

where $\Gamma$ is one of the 16 independent $\gamma$-matrix combinations. We shall be concerned with constructing operators with a given set of quantum numbers by taking linear combinations with different paths and different expressions for $\Gamma$.

For orientation, we first consider the case where there is no path $\mathrm{P}$ and the local operator has

$x_{1}=x_{2}$. Then in the continuum such a bilinear can have $J^{P C}$ values as listed. The historic name of the isovector non-strange meson with those quantum numbers is also added for convenience. 


$\begin{array}{ccc}\Gamma & J^{P C} & \text { name } \\ 1 & 0^{++} & \delta \\ \gamma_{4} & 0^{+-} & \text {exotic } \\ \gamma_{i} & 1^{--} & \rho \\ \gamma_{i} \gamma_{4} & 1^{--} & \rho \\ \gamma_{5} & 0^{-+} & \pi \\ \gamma_{5} \gamma_{4} & 0^{-+} & \pi \\ \gamma_{i} \gamma_{j} & 1^{+-} & \mathrm{B} \\ \gamma_{5} \gamma_{i} & 1^{++} & \mathrm{A}_{1}\end{array}$

Note that the non-relativistic limit of these 16 bilinears, which corresponds to projecting the quark spinor with $1+\gamma_{4}$, leaves 4 combinations which have the $\pi$ and $\rho$ quantum numbers only - as in the naive quark model. Note also that when the quark and antiquark have different masses, the meson will not be an eigenstate of charge conjugation $\mathrm{C}$. Thus, for example, the $1^{+-}$and $1^{++}$axial mesons will mix.

The general case will involve discussion of the properties of the path $\mathrm{P}$ under rotations, reflections and charge conjugation. Since the spinor structure is in a fixed basis, it is sufficient to consider separately the transformation properties of the path $\mathrm{P}$ and then combine the result with that given above for the quark bilinears. With this in mind, we now consider the classification of the path $\mathrm{P}$ under the symmetries of the lattice: rotation, reflection, translation and charge conjugation.

\section{A.1 Discrete group theory}

The group of rotations and inversions for a three dimensional spatial lattice is the cubic group $O_{h}$. This is thus the appropriate classification group for bosonic transfer matrix eigenstates with momentum zero. For non-zero momentum the space group needs to be used instead - see ref [16].

Here we gather together some of the appropriate properties of this group and its representations. The representations are labelled by parity $\mathrm{P}$ and charge conjugation $\mathrm{C}$ in the same way as bosonic representations of the $\mathrm{SU}(2)$ rotation group appropriate to the continuum formulation. Thus the essential difference between the lattice eigenstates and continuum eigenstates lies in the 'spin'.

Whereas a state of spin $J$ has $2 J+1$ spin components which are degenerate in mass and form a $2 J+1$ dimensional representation in the continuum, for $O_{h}$ there exist only 1,2 and 3 -dimensional representations. The 1-dimensional representations are labelled $A_{1}$ and $A_{2}$, there is a 2-dimensional $E$ representation and the 3-dimensional representations are $T_{1}$ and $T_{2}$.

The relationship of these representations to those of $\mathrm{SU}(2)$ can be derived by restricting the 
$\mathrm{SU}(2)$ representations to the rotations allowed by cubic symmetry and classifying them under $O_{h}$. This process (called subducing) yields the results (tabulated to $J=4$ ):

$$
\begin{array}{lc}
J=0 & A_{1} \\
J=1 & T_{1} \\
J=2 & E T_{2} \\
J=3 & A_{2} T_{1} T_{2} \\
J=4 & A_{1} E T_{1} T_{2}
\end{array}
$$

Thus, for example, as the lattice spacing is decreased, a $J=2$ state will be recognised by degenerate masses in $E$ and $T_{2}$ representations. This is indeed observed in glueball studies [18, 17. Conversely, a state observed in the $T_{2}$ representation on a lattice could have $J=2$ or 3 and this choice can only be resolved by observing the degenerate partners (e.g. $E$ for $J=2$ or $A_{2}$ and $T_{1}$ for $J=3$ ). In this way, with sufficiently accurate lattice spectra in all $O_{h}$ representations, the rotationally invariant continuum levels can be constructed for any $J$ value in principle.

Optimal signal to noise comes from constructing operators on a lattice which create states of given $O_{h}$ representation. This has been well documented in glueball studies where the appropriate observables are closed Wilson loops - see ref [19]. In the present study, the appropriate lattice constructs are the paths $\mathrm{P}$ from $x_{1}$ to $x_{2}$ along the links of the lattice. These paths must then be classified according to the representations of the cubic group $O_{h}$. The most powerful method is to use the projection table [19] which directly gives the relevant combination of paths with different cubic rotations for each representation.

As well as the spatial path $\mathrm{P}$, the mesonic operator will have a spin component coming from the quark spinors. On a lattice, where the spatial symmetry is $O_{h}$, the only change in the classification of these bilinears is that the singlets $(J=0)$ become $A_{1}$ while the triplets $(J=1)$ become $T_{1}$. This change has one implication which has not been discussed before. The ' $\rho$ ' bilinear on a lattice is in the $T_{1}$ representation and so allows $J=1$ and 3 . Thus the excited state of the $\rho$ meson seen in lattice studies could be the $\mathrm{g}\left(3^{--}\right)$rather than a heavier $\rho\left(1^{--}\right)$as usually assumed.

We need to combine the $O_{h}$ representation of this quark bilinear with that of the spatial path $\mathrm{P}$ (from $x_{1}$ to $x_{2}$ ). The combined operator thus will lie in a representation given by the Clebsch-Gordan decomposition of the product of representations. When the quark bilinear is in the trivial $A_{1}$ representation, this is straightforward since the final representation is just that of the spatial path. For the $T_{1}$ representation of quark bilinears, we need the decomposition:

$$
\begin{aligned}
& T_{1} \otimes A_{1}=T_{1} \\
& T_{1} \otimes A_{2}=T_{2} \\
& T_{1} \otimes E=T_{1} \oplus T_{2}
\end{aligned}
$$




$$
\begin{aligned}
& T_{1} \otimes T_{1}=A_{1} \oplus T_{1} \oplus T_{2} \oplus E \\
& T_{1} \otimes T_{2}=A_{2} \oplus T_{1} \oplus T_{2} \oplus E
\end{aligned}
$$

As well as the Clebsch-Gordan decomposition, we will need the Clebsch-Gordan coefficients to construct the explicit operators required. These will be given later for the cases of most interest.

Since the general path with no symmetry will contribute to all $O_{h}$ representations in principle, one can create operators with all quantum numbers. Since it is practical to start with simpler cases, we now discuss the states that can be constructed from more symmetric paths: straight, L-shaped and U-shaped.

\section{A.2 Orbitally excited mesons.}

A full study of mesons with orbital angular momentum needs non-local operators. The simplest case to consider is that of a straight line from $x_{1}$ to $x_{2}$ joining the quark and antiquark. If this straight line is along the lattice axis then the result is that there are $A_{1}, T_{1}$ and $E$ representations. In a notation where the directed path is labelled by the axis of its orientation these representations are given explicitly by:

$$
\begin{array}{cc}
A_{1}^{++} & s_{1}+s_{2}+s_{3} \\
T_{1}^{--} & p_{1}, p_{2}, p_{3} \\
E^{++} & s_{1}-s_{2}, 2 s_{3}-s_{1}-s_{2}
\end{array}
$$

where $p_{j}=(j-\bar{j})$ and $s_{j}=(j+\bar{j})$ and where $\overline{1}$ is the path directed along the negative $x$-axis, for example. The conventional normalisation factors have been omitted to simplify the notation.

These three representations correspond partly to the $S$-, $P$ - and $D$-wave orbital excitations of the naive quark model. The path combinations given play the role of the spherical harmonics in $\mathrm{SU}(2)$. A discussion of the construction of operators in the $T_{1}^{--}$representation has also been given by ref. [5] in their study of $P$-wave states.

Consider now the states that can be constructed by combining these path representations with the quark bilinears. For the 'large components' which survive in the non-relativistic limit, the bilinears are in representations $A_{1}^{-+}$and $T_{1}^{--}$. Combining these with the above straight-path representations yields: 


$\begin{array}{ccccc}\bar{q} \Gamma q & \text { Path } & \text { State } & \text { operator } & J^{P C} \\ & & & \gamma_{5}\left(s_{1}+s_{2}+s_{3}\right) & 0^{-+}, 4^{-+} \\ A_{1}^{-+} & A_{1}^{++} & A_{1}^{-+} & \gamma_{j}\left(s_{1}+s_{2}+s_{3}\right) & 1^{--}, 3^{--} \\ T_{1}^{--} & A_{1}^{++} & T_{1}^{--} & & \\ & & & \gamma_{5} p_{j} & 1^{+-}, 3^{+-} \\ A_{1}^{-+} & T_{1}^{--} & T_{1}^{+-} & \gamma_{1} p_{1}+\gamma_{2} p_{2}+\gamma_{3} p_{3} & 0^{++}, 4^{++} \\ T_{1}^{--} & T_{1}^{--} & A_{1}^{++} & \gamma_{k} p_{i}-\gamma_{i} p_{k} & 1^{++}, 3^{++} \\ T_{1}^{--} & T_{1}^{--} & T_{1}^{++} & \gamma_{k} p_{i}+\gamma_{i} p_{k} & 2^{++}, 3^{++} \\ T_{1}^{--} & T_{1}^{--} & T_{2}^{++} & & 2^{++}, 4^{++} \\ T_{1}^{--} & T_{1}^{--} & E^{++} & \gamma_{1} p_{1}-\gamma_{2} p_{2}, 2 \gamma_{3} p_{3}-\gamma_{1} p_{1}-\gamma_{2} p_{2} & 2^{++} \\ & & & \gamma_{5}\left(s_{1}-s_{2}\right), \gamma_{5}\left(2 s_{3}-s_{1}-s_{2}\right) & 2^{-+}, 4^{-+} \\ A_{1}^{-+} & E^{++} & E^{-+} & \gamma_{j}\left(2 s_{j}-s_{i}-s_{k}\right) & 1^{--}, 3^{--} \\ T_{1}^{--} & E^{++} & T_{1}^{--} & \gamma_{j}\left(s_{i}-s_{k}\right) & 2^{--}, 3^{--} \\ T_{1}^{--} & E^{++} & T_{2}^{--} & & \end{array}$

where $i, j, k$ are cyclic and there is no implied summation. The two lowest $J$-values corresponding to the $O_{h}$ representation are also given for convenience. The above operators have the same quantum numbers when multiplied by $\gamma_{4}$, indeed projection with $\left(1+\gamma_{4}\right) / 2$ is useful to reduce computation. Note that while operators with different quantum numbers are orthogonal to each other, the two $T_{1}^{--}$operators will mix through spin-orbit interactions.

Note that with an isotropic spatial distribution, the quark bilinear can create, through the 'small' spinor components, the $P$-wave states $A_{1}^{++}, T_{1}^{+-}$and $T_{1}^{++}$(particles $\delta, \mathrm{B}$ and $\mathrm{A}_{1}$ ). These extra operators allow a fuller variational basis to be used in the study of these particular states.

The lowest $J^{P C}$ states created are just those of the continuum $L$-excited naive quark model for $S$ - and $P$-waves with an incomplete $D$-wave in the sense that the $D$-wave mesons with $J^{P C}=2^{--}$and $3^{--}$will both be created by the $T_{2}^{--}$operator above. In order to separate these states, one needs to use operators in the $E^{--}$and $A_{2}^{--}$representations. One way in which these are accessible is by using a 'straight diagonal path' from quark to antiquark. Such a path is the sum of the two L-shaped paths going to the far corner of a square via the sides. It acts, in some way, as a lattice opportunity to study operators at $45^{0}$ to the lattice axes and so helps to explore higher spin. The 12 such paths contribute to the $A_{1}^{++}, E^{++}, T_{2}^{++}, T_{2}^{--}, T_{1}^{--}$representations. Using a notation $[i \bar{j}]$ to describe such a path to the $(i,-j)$ diagonal corner from the origin etc., then appropriate combinations for the $T_{2}^{++}$spatial operator are

$$
t_{k}=[i j]-[i \bar{j}]-[j \bar{i}]+[\bar{i} \bar{j}]
$$

with $i, j, k$ cyclic. Combining this with the $T_{1}^{--}$quark bilinear gives the required operators: 


$\begin{array}{ccccc}\bar{q} \Gamma q & \text { Path } & \text { State } & \text { operator } & J^{P C} \\ & & & & \\ T_{1}^{--} & T_{2}^{++} & A_{2}^{--} & \gamma_{1} t_{1}+\gamma_{2} t_{2}+\gamma_{3} t_{3} & 3^{--}, 5^{--} \\ T_{1}^{--} & T_{2}^{++} & E^{--} & \gamma_{1} t_{1}-\gamma_{2} t_{2}, 2 \gamma_{3} t_{3}-\gamma_{1} t_{1}-\gamma_{2} t_{2} & 2^{--}, 4^{--} \\ T_{1}^{--} & T_{2}^{++} & T_{1}^{--} & \gamma_{i} t_{k}+\gamma_{k} t_{i} & 1^{--}, 3^{--} \\ T_{1}^{--} & T_{2}^{++} & T_{2}^{--} & \gamma_{i} t_{k}-\gamma_{k} t_{i} & 2^{--}, 3^{--}\end{array}$

This illustrates that the $2^{--}$and $3^{--}$states can be separated and thus explored from these operators. This discussion of straight paths completes the construction of operators which allow a full study of the $D$-wave mesons. Our results for the lattice operators needed to construct a $D$-wave $\rho$ meson are similar to those presented in ref [11].

It is possible to study some aspects of $F$-wave mesons on the lattice fairly simply. For example, consider the 'straight cubic diagonal' paths which go from the origin to the corners of a cube centered at the origin. Paths with this symmetry can be defined on a lattice as a sum over the six equivalent shortest routes to a corner along the links in each case. From such path combinations, the $A_{2}^{--}$representation can be constructed as a sum of these paths to each of the 8 corners of the cube with a sign that alternates between adjacent corners. This represents a spatial $L=3$ excitation. When combined with the $A_{1}^{-+}$quark bilinear, this yields a $A_{2}^{+-}$state which enables study of the $3^{+-}$F-wave spin-singlet meson.

\section{A.3 Hybrid and exotic $J^{P C}$ states}

Hybrid mesons are defined as having the gluonic field excited in a non-trivial way. In the case of static quarks, this is a very clear prescription and a thorough study has been made of the excited gluonic energy levels [2]. Solving for the meson spectrum in this excited static potential in the adiabatic approximation then gives 8 degenerate lowest lying hybrid states. One of the consequences of these excited gluonic modes is that hybrid mesons can have $J^{P C}$ values not allowed to mesons which have their gluonic degrees of freedom in the ground state. We are particularly interested in constructing mesonic operators with $J^{P C}$-values which are not present in the naive quark model since these have a clear signature experimentally. These have exotic quantum numbers which are $0^{--}, 0^{+-}, 1^{-+}, 2^{+-}$, etc. Because of the many-to-one map from $O_{h}$ representation to $J^{P C}$, it is necessary in principle to study all $O_{h}$ representations as discussed above. Nevertheless, promising representations to study are $A_{1}^{--}, A_{1}^{+-}, T_{1}^{-+}$and $E^{+-}$since they only have a non-exotic contribution at $J$-values 3 or more higher than the exotic one unlike the case of the $T_{2}^{+-}$representation where the exotic $J=2$ state may be contaminated by contributions from a non-exotic $J=3$ meson.

For straight paths, none of the states in the table above are of the required exotic representation. This is to be expected since the straight path corresponds to the naive $L$-excited quark model and so should not generate exotic $J^{P C}$ states. The exception is that there are relativistic couplings to quark bilinears which do allow exotic representations. If one has in mind the 
picture of a hybrid state with exotic quantum numbers as arising from gluonic excitations, then such operators with no gluonic excitation are not particularly promising in the sense that the coupling may be very small and hence swamped by noise in practice.

The more direct way to create exotic states is to use a path from $x_{1}$ to $x_{2}$ which is not straight. The evidence from a lattice study of hybrid mesons formed from static quarks [2] is that the lowest energy excitation comes from U-shaped paths. In our case, a U-shaped path will have 24 different orientations and will contribute to the following representations: $A_{1}^{++}, A_{2}^{++}, E^{++}$ (twice), $T_{1}^{--}, T_{1}^{+-}, T_{1}^{-+}, T_{2}^{--}, T_{2}^{+-}, T_{2}^{-+}$. Combining these spatial representations with the quark bilinears $A_{1}^{-+}$and $T_{1}^{--}$then gives access to most of the exotic states discussed above as well as many copies of the non-exotic ones.

The static quark results show [2] that the lowest energy hybrid states come from an operator which is the difference of U-shaped paths from quark to antiquark of the form $\sqcap-\sqcup$. We find that in this study also, exotic states are only created from this difference of paths and so we specialise to this case. The $k$-component of the representation of the spatial paths is given by the following (where $i j$ refers to the path combination $\sqcap-\sqcup$ from $x_{1}$ to $x_{2}$ along the $i$-axis with transverse extent in the $j$-direction);

$$
\begin{array}{lc}
T_{1}^{+-} & u_{k}=i j-j i-\bar{i} j+\bar{j} i \\
T_{1}^{-+} & v_{k}=i k+\bar{i} k+j k+\bar{j} k \\
T_{2}^{+-} & U_{k}=i j+j i-\bar{i} j-\bar{j} i \\
T_{2}^{-+} & V_{k}=i k+\bar{i} k-j k-\bar{j} k
\end{array}
$$

with $i, j, k$ cyclic. The $T_{1}$ spatial path combinations are illustrated here:
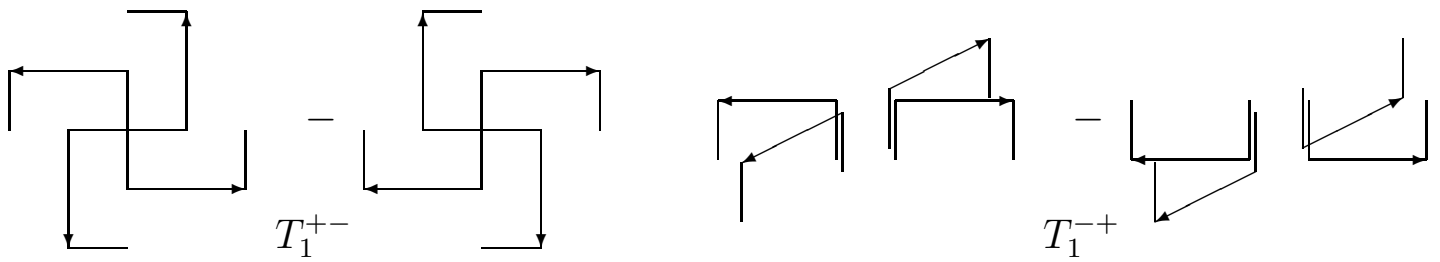

The $T_{1}$ spatial representations correspond to $L=1$ and so should lie lower in energy than the $T_{2}$ cases. Thus we expect the lowest lying hybrid mesons to be obtained by combining the $T_{1}$ spatial behaviour with the quark bilinear. This gives the operators shown in Table 3 . The mesonic quantum numbers of these hybrid states are exactly the same as those produced by the lowest gluonic excitation for static quarks [2]. This includes most of the exotic possibilities.

As a further support for this identification of the lowest lying hybrids, we can compare with hybrid operators constructed using L-shaped paths instead of U-shaped. Considering this time the difference of the two L-shaped paths to the opposite corner of a square via the edges, this combination contributes to $E^{+-}, A_{2}^{+-}, T_{1}^{+-}, T_{1}^{-+}, T_{2}^{-+}$spatial representations. This set of hybrid spatial excitations is very similar to that from U-shaped paths. In particular the excitations of lowest $L\left(T_{1}\right.$ with $\left.L=1\right)$ are the same. For completeness we give the construction 


$\begin{array}{ccccc}\bar{q} \Gamma q & \text { Path } & \text { State } & \text { operator } & J^{P C} \\ & & & & \\ A_{1}^{-+} & T_{1}^{+-} & T_{1}^{--} & \gamma_{5} u_{j} & 1^{--}, 3^{--} \\ T_{1}^{--} & T_{1}^{+-} & A_{1}^{-+} & \gamma_{1} u_{1}+\gamma_{2} u_{2}+\gamma_{3} u_{3} & 0^{-+}, 4^{-+} \\ T_{1}^{--} & T_{1}^{+-} & E^{-+} & \gamma_{1} u_{1}-\gamma_{2} u_{2}, 2 \gamma_{3} u_{3}-\gamma_{1} u_{1}-\gamma_{2} u_{2} & 2^{-+}, 4^{-+} \\ T_{1}^{--} & T_{1}^{+-} & T_{1}^{-+} & \gamma_{k} u_{i}-\gamma_{i} u_{k} & 1^{-+}, 3^{-+} \\ T_{1}^{--} & T_{1}^{+-} & T_{2}^{-+} & \gamma_{k} u_{i}+\gamma_{i} u_{k} & 2^{-+}, 3^{-+} \\ & & & & \\ A_{1}^{-+} & T_{1}^{-+} & T_{1}^{++} & \gamma_{5} v_{j} & 1^{++}, 3^{++} \\ T_{1}^{--} & T_{1}^{-+} & A_{1}^{+-} & \gamma_{1} v_{1}+\gamma_{2} v_{2},+\gamma_{3} v_{3} & 0^{+-}, 4^{+-} \\ T_{1}^{--} & T_{1}^{-+} & E^{+-} & \gamma_{1} v_{1}-\gamma_{2} v_{2}, 2 \gamma_{3} v_{3}-\gamma_{1} v_{1}-\gamma_{2} v_{2} & 2^{+-}, 4^{+-} \\ T_{1}^{--} & T_{1}^{-+} & T_{1}^{+-} & \gamma_{k} v_{i}-\gamma_{i} v_{k} & 1^{+-}, 3^{+-} \\ T_{1}^{--} & T_{1}^{-+} & T_{2}^{+-} & \gamma_{k} v_{i}+\gamma_{i} v_{k} & 2^{+-}, 3^{+-}\end{array}$

Table 3: Hybrid meson operators, where $i, j, k$ are cyclic and factors of $\gamma_{4}$ can be included too.

of these hybrid spatial paths, using a notation that the L-shaped path first in the $i$-direction and then in the $j$-direction is $(i j)$ with $\bar{i}$ representing the negative direction etc., with $i, j, k$ cyclic:

$$
\begin{array}{lc}
T_{1}^{+-} & (i j)-(j i)+(j \bar{i})-(\bar{i} j)+(\overline{i j})-(\overline{j i})+(\overline{j i})-(i \bar{j}) \\
T_{1}^{-+} & (k i)+(k \bar{i})+(k j)+(k \bar{j})-(\bar{k} i)-(\bar{k} \bar{i})-(\bar{k} j)-(\bar{k} \bar{j}) \\
& +(i \bar{k})+(\bar{i} \bar{k})+(j \bar{k})+(\bar{j} \bar{k})-(i k)-(\bar{i} k)-(j k)-(\bar{j} k)
\end{array}
$$

One exotic state which is of particular interest is the $A_{1}^{+-}$state since it can also be created from an isotropic spatial construction by a quark bilinear using $\Gamma=\gamma_{4}$. Thus one can explore its creation and annihilation using either the isotropic operators (local or $S$-wave) or the non-local operators (U or L-shaped). Again the isotropic spatial construction does not have an excited gluonic field and so may not give good signal to noise for an exotic hybrid meson.

Only one exotic representation $\left(A_{1}^{--}\right)$cannot be obtained using the paths discussed above. To create this state needs paths with even less symmetry than the planar U and L-shaped cases we have discussed in detail. Because such a complex path shape is necessary, one expects that the state will be relatively high lying in mass.

For completeness, we point out that gluonic excitations of light quarks can be studied in principle in the limit $x_{1} \rightarrow x_{2}$ where only local quark propagators will be needed. The above U- and L-shaped paths are then not appropriate and the simplest choice is a square path with one corner at the quark and antiquark source. The construction of combinations of such paths in appropriate representations of the cubic group is discussed in ref [12] where they were used with a static adjoint colour source. Here the same construction is needed and the quark and 
antiquark at $x_{1}=x_{2}$ are joined by the square paths. The lowest gluonic excitations are expected to come from the $T_{1}^{+-}$and $T_{1}^{-+}$spatial operators again - just as for $x_{1} \neq x_{2}$. These spatial operators are then to be combined with quark bilinears just as above.

\section{A.4 Mesonic correlations.}

The mesonic operators we have discussed are of the form

$$
\bar{\psi}_{2}\left(x_{2}\right) P\left(x_{2}, x_{1}\right) \Gamma \psi_{1}\left(x_{1}\right)
$$

where $\Gamma$ is one of the 16 independent $\gamma$-matrix combinations and $P$ is a product of links. The subscripts on the spinors refer to the possibility of different masses for quark and antiquark. A sum over $x_{1}$ and $x_{2}$ with appropriate path combinations is needed to project out the quantum numbers of interest as discussed above. We consider the operator defined in a time slice at time $t$.

The mesonic state can be explored on a lattice by measuring the correlation of this operator at $t=0$ with a similar operator at time $t$. Allowing for different operators at source $(\mathrm{A}, x$ with $t=0)$ and sink (B, $y$ with time $t$ ) then gives the correlation to be measured

$$
C_{B A}(t)=<\bar{\psi}_{1}\left(y_{1}\right) \gamma_{4}\left(P^{B} \Gamma^{B}\right)^{\dagger} \gamma_{4} \psi_{2}\left(y_{2}\right) \bar{\psi}_{2}\left(x_{2}\right) P^{A} \Gamma^{A} \psi_{1}\left(x_{1}\right)>
$$

In the quenched approximation, for a Wilson-like fermionic action, the quark propagators $S$ can be explicitly introduced to re-express this as

$$
C_{B A}(t)=-<\gamma_{5} S_{1}^{\dagger}\left(y_{1}, x_{1}\right) \gamma_{5} \gamma_{4}\left(P^{B} \Gamma^{B}\right)^{\dagger} \gamma_{4} S_{2}\left(y_{2}, x_{2}\right) P^{A} \Gamma^{A}>
$$

For $\mathrm{A}=\mathrm{B}$, this correlation is positive definite.

This expression for the correlation has the colour structure of two propagators connected by paths $\mathrm{P}^{A}$ and $\mathrm{P}^{B}$.

The symmetries of the lattice can be used to increase the precision of a measurement by combining it with the correlation measured on lattices reflected in the time or space directions using the identity for the propagators that

$$
S(x, y ; U)=\gamma_{i} \gamma_{5} S\left(x^{I}, y^{I} ; U^{I}\right) \gamma_{5} \gamma_{i}
$$

where the superscript $I$ refers to the lattice coordinates and links after inversion in the $i$ direction (where $i=1,2,3$ or 4 ). Charge conjugation provides another relation since

$$
S(x, y ; U)=\gamma_{4} \gamma_{2} \gamma_{5} S^{*}\left(x, y ; U^{*}\right) \gamma_{5} \gamma_{2} \gamma_{4}
$$

Note that the identity for inversion with $i=4$ can be used to combine measurements of the correlation at $t= \pm|t|$ using $C_{B A}(-t)= \pm C_{B A}(t)$ with the appropriate sign coming from commuting $\gamma_{4} \gamma_{5}$ past $\Gamma^{A}$ and $\Gamma^{B}$. 
In practice we construct the sink operator (B) to have the required quantum numbers but employ a source operator (A) which has less symmetry but a non-zero overlap with the sink operator. In this situation, it is useful to use the symmetries of the lattice under space reflections to eliminate terms in the correlation which have zero expectation value. This reduces the statistical noise of the measurement.

Note that for the U-shaped paths, even though we use a fixed source direction, we measure correlations of source and sink operators for 64 different combinations of orientations and gamma matrices. After averaging equivalent cases, these correlations allow a reconstruction of the mesonic quantum numbers of the hybrid states tabulated above.

\section{References}

[1] C. Michael, Phys. Rev. Lett. 56 (1986) 1219; A. Huntley and C. Michael, Nucl. Phys. B286 (1987) 211.

[2] L.A. Griffiths, C. Michael and P.E.L. Rakow, Phys. Lett. 129B (1983) 351; S. J. Perantonis and C. Michael, Nucl. Phys. B347 (1990) 854.

[3] P. Bacilieri et al., Phys. Lett. 214B (1988) 115; Nucl. Phys. B317 (1989) 509.

[4] P. Lacock and C. Michael, (UKQCD Collaboration) Phys. Rev. D 52 (1995) 5213.

[5] M. Wingate et al., Phys. Rev. D52 (1995) 307.

[6] T.A. DeGrand and M.W. Hecht, Phys. Rev. D 46 (1992) 3937; Phys. Lett. 275B (1992) 435.

[7] M.W. Hecht et al., Phys. Rev. D 47 (1993) 285.

[8] S. J. Perantonis, A. Huntley and C. Michael, Nucl. Phys. B326 (1989) 544.

[9] P. Lacock et al., (UKQCD Collaboration) Phys. Rev. D 51 (1995) 6403.

[10] C. Michael and H. Shanahan, (UKQCD Collaboration), Contribution to Lattice '95, heplat/9508030.

[11] R. Gupta, D. Daniel and J. Grandy, Phys. Rev. D 48 (1993) 3330.

[12] I.H. Jorysz and C. Michael, Nucl. Phys. B302 (1988) 448.

[13] C. Michael and A. McKerrell, Phys. Rev. D 51 (1995) 3745.

[14] Y. Kuramashi et al., Phys. Rev. Lett. 72 (1994) 3448.

[15] F. E. Close, Proc. Hadron Conference, Manchester 1995; hep-ph/9509245. 
[16] J. Cornwell, Group Theory in Physics, Vol. 1 (Academic Press), London, 1984.

[17] C. Michael and M. Teper, Nucl. Phys. B314 (1989) 347.

[18] C. Michael, G. A. Tickle and M. J. Teper, Phys. Lett. 207B (1988) 313.

[19] C. Michael, Acta Phys. Polonica B21 (1990) 119. 